\title{
Assessment of the Effectiveness of Various Adult Mosquito Sampling Methods in a Thickly Populated Urban Slum Settlement - A study from Besant Nagar, Chennai, India
}

\section{Sangamithra Ravishankaran}

National Institute of Malaria Research

\section{Aswin Asokan}

National Institute of Malaria Research

Johnson Amala Justin N A

Ministry of Health and Family Welfare

Shalu Thomas

Madras Christian College

Vasna Joshua

National Institute of Epidemiology

Manu Thomas Mathai

Madras Christian College

Alex Eapen ( $\sim$ alexeapen@yahoo.com )

National Institute of Malaria Research

\section{Research}

Keywords: CDC-Light trap, Urban slum, Anopheles stephensi, Resting collection, Vector incrimination

Posted Date: July 10th, 2020

DOI: https://doi.org/10.21203/rs.3.rs-30372/v2

License: (9) (i) This work is licensed under a Creative Commons Attribution 4.0 International License. Read Full License 
The authors have withdrawn this preprint from Research Square 